\title{
Multilinguales
}

\section{La langue maternelle, une stratégie pour enseigner/apprendre la langue étrangère}

The mother tongue, a strategy for teaching/learning foreign language

\section{Rabéa Benamar}

\section{(2) OpenEdition \\ 1 Journals}

\section{Édition électronique}

URL : http://journals.openedition.org/multilinguales/1632

DOI : 10.4000/multilinguales. 1632

ISSN : 2335-1853

Éditeur

Université Abderrahmane Mira - Bejaia

\section{Édition imprimée}

Date de publication : 1 juin 2014

Pagination : 139-158

ISSN : 2335-1535

\section{Référence électronique}

Rabéa Benamar, "La langue maternelle, une stratégie pour enseigner/apprendre la langue étrangère », Multilinguales [En ligne], 3 | 2014, mis en ligne le 03 juin 2014, consulté le 17 septembre 2019. URL : http://journals.openedition.org/multilinguales/1632; DOI : 10.4000/multilinguales.1632

Ce document a été généré automatiquement le 17 septembre 2019.

\section{(†) $\ominus$

Multilinguales est mise à disposition selon les termes de la Licence Creative Commons Attribution Pas d'Utilisation Commerciale - Pas de Modification 4.0 International 


\title{
La langue maternelle, une stratégie pour enseigner/apprendre la langue étrangère
}

The mother tongue, a strategy for teaching/learning foreign language

\author{
Rabéa Benamar
}

1 La langue maternelle (désormais LM) ${ }^{1}$ est caractérisée par le fait qu'elle est acquise de façon naturelle. Selon Vygotski (1985), l'apprentissage de la LM s'opère de manière inconsciente et elle est acquise par l'enfant spontanément à travers des expériences provoquées par le contact avec son environnement immédiat. Alors que l'apprentissage d'une langue étrangère (désormais LE) commence par la prise de conscience et l'existence d'une intention.

2 Ainsi, apprendre une LE est un processus qui sollicite plus d'efforts que l'apprentissage d'une langue maternelle qui, au contraire, est un fait plus naturel se constituant quasi spontanément. La particularité des interactions, en classe de LE, est que la langue est à la fois l'outil de transmission des savoirs et l'objet même de l'enseignement/ apprentissage de cette langue. En apprenant la LE, l'élève découvre un autre système linguistique et une autre culture. Le caractère scolaire de l'apprentissage d'une LE présuppose des difficultés quant à son enseignement/apprentissage. Il devient alors impératif de prendre en compte toutes les données relatives à ce dernier.

3 En ce sens, nous distinguerons, comme le font R. Porquier et B. Py (2004:59) dans le domaine de l'appropriation d'une langue, deux niveaux d'interaction: le contexte macro qui renvoie aux déterminations sociales au sens le plus large, et le contexte micro, qui dans notre cas correspondrait à la salle de classe. Nous pouvons appliquer cette distinction à notre étude dans la mesure où nous pensons que le premier contexte peut influencer le second. En Algérie, l'enseignement/apprentissage du français langue étrangère (désormais $\mathrm{FLE}$ ), se déroule dans un contexte sociolinguistique, institutionnel, historique, etc. particulier. La spécificité de ce contexte linguistique engendre inévitablement des phénomènes particuliers (comme l'alternance codique). Cependant, certains ${ }^{2}$ voient dans la LM une source de difficultés et d'erreurs. Pourtant, 
sur le terrain, il en est autrement, où enseignants et apprenants recourent souvent à la LM. En fait, c'est une pratique naturelle conforme à toute situation de communication de contact de langues (Causa, 2002).

4 Ceci nous amène à nous poser la problématique suivante : l'alternance codique ${ }^{3}$ peutelle avoir un rôle stratégique en classe de FLE?

5 Nous postulons, dans le cadre de cette recherche, que l'alternance codique est une stratégie de communication et que le recours à la LM permet de maximiser l'efficacité de la communication aussi bien du côté de l'élève, pour qui elle peut servir de « langue d'appui » (Castellotti, 2001) que du côté de l'enseignant. Nous adhérons à l'idée que les ressources langagières multiples des interactants pourraient être mises à profit en classe de FLE.

6 Pour vérifier notre hypothèse, nous distinguerons entre alternances relais et alternances tremplins (Moore, 1996) et nous nous appuierons sur les stratégies proposées par M. Causa (2002) ainsi que sur les différentes catégories d'alternances codiques, selon leur fonction, recensées par M. Cambra Giné (2003).

7 L'objectif de notre étude est de connaître les raisons du changement de code des interactants en classe de FLE. Une étude qui se veut empirique et fondée principalement sur des enregistrements audio ${ }^{4}$ complétés par des entretiens (semidirectifs) avec des enseignants et quelques élèves de classe terminale ${ }^{5}$.

8 Le répertoire verbal de ces élèves est constitué de l'arabe dialectal (langue maternelle), de l'arabe standard (langue d'étude), du français (première langue étrangère) et de l'anglais (deuxième langue étrangère). L'enseignant possède aussi la même langue maternelle que les élèves mais nous le considérons comme étant un expert dans la pratique du français de par sa formation et de par son statut.

9 Ainsi, nous considérons la classe comme une situation de communication potentiellement bilingue dans laquelle l'enseignant est perçu comme un locuteur bilingue et les apprenants comme des bilingues virtuels (Causa, 1996).

10 Dans cette perspective et dans un premier temps, nous ferons un tour d'horizon de cette situation langagière si spécifique au contexte d'enseignement / apprentissage du FLE en Algérie et dans un second temps, nous définirons la notion d'alternance codique, objet de notre analyse.

\section{Langues en présence en Algérie}

11 Le paysage sociolinguistique algérien se caractérise par l'existence de plusieurs langues; ce qui illustre bien l'hétérogénéité du pays. Ainsi, dans cet espace, plusieurs langues coexistent, se rencontrent et se mélangent à savoir l'arabe classique (ou standard), différents dialectes (arabe algérien et berbère) et le français.

$12 *^{*}$ L'arabe classique $^{6}$ : langue nationale et officielle, c'est une langue qui a tendance à s'imposer dans l'administration, l'enseignement, etc. Elle n'est pas utilisée dans la communication quotidienne mais apprise à l'école. Ainsi, une fois arrivé en première année primaire, l'élève algérien découvre l'arabe classique ou standard ${ }^{7}$.

13 * L'arabe dialectal : ou darija ${ }^{8}$ langue essentiellement orale avec, certes, des variantes selon les régions du pays ${ }^{9}$. Elle est considérée comme étant la LM de la majorité de la population algérienne. C'est donc la langue de la première socialisation car c'est à 
travers elle que se forge l'imaginaire de l'individu et son univers affectif, pour la majorité des Algériens (K.Taleb Ibrahimi, $1997: 28$ ).

$14{ }^{*}$ Le berbère ou le tamazight : il se décline en plusieurs variantes. Il est parlé par les Kabyles et les Chaouis au nord de l'Algérie et les Mozabites et les Touaregs au sud. Depuis 2002, le tamazight est officiellement «langue nationale » et enseigné dans les établissements scolaires des zones berbérophones.

15 * Le français: la langue française est une langue étrangère au statut privilégié (Queffélec, 2002) dans la société algérienne, et ceci à différents niveaux : économique, social, éducatif, etc. Dans le système éducatif, le français est enseigné à partir de la troisième année primaire, alors que l'anglais ne l'est qu'à partir de la première année $\mathrm{du}$ collège. Notons aussi qu'à l'université, la langue française est la langue d'enseignement dans certaines filières.

G. Grandguillaume (1983) résume ainsi la situation des langues en présence en Algérie :

«Trois langues sont utilisées: la langue arabe, la langue française et la langue maternelle. Les deux premières sont des langues de culture, de statut écrit. Le français est aussi pratiqué comme langue de conversation.

Toutefois, la langue maternelle, véritablement parlée dans la vie quotidienne, est toujours un dialecte, arabe ou berbère. » (p. 11)

17 Ainsi, cette situation linguistique peut avoir des conséquences sur les pratiques langagières des élèves en classe de langue. En effet, dans cet espace plus restreint qu'est l'école, cette cohabitation linguistique fait que la classe de LE représente une situation de communication de contact de langues par excellence (Causa, 2002) ; autrement dit, une situation dans laquelle enseignant et/ou apprenant peuvent être amenés à utiliser deux langues : la langue étrangère et la langue maternelle.

Cela dit, nous pensons que dans une situation de communication caractérisée par un contact de langues, il y a forcément des répercussions au niveau de l'enseignement/ apprentissage en général, et de LE en particulier, pour nous le français. En effet, cette situation particulière n'exclut pas d'éventuelles marques transcodiques ${ }^{10}$ repérables dans le discours en classe de FLE. Leurs utilisations peuvent signaler le besoin de résoudre un problème et peuvent se traduire de différentes façons: emprunts, interférences, alternance codique, etc. ${ }^{11}$

\section{L'alternance codique}

19 Certaines recherches en analyse conversationnelle (cf. entre autres Lüdi \& Py, 2003) ont montré, qu'en contexte exolingue ${ }^{12}$, les interlocuteurs mettent en œuvre des stratégies pour combler le déséquilibre au niveau des compétences langagières. Nous entendons par « stratégie » au sens de M. Causa (2002) :

«L'ensemble des actions dirigées par les sujets communicants pour atteindre l'accomplissement d'une tâche globale visant à la transmission/à l'appropriation des données en langue cible et, en même temps, à la résolution de problèmes communicatifs qui tiennent au déséquilibre des compétences en langue cible chez les acteurs de l'espace-classe » (p. 57).

Ce qui suppose que l'on peut identifier des stratégies aussi bien du côté de l'enseignant (stratégies communicatives d'enseignement) que du côté de l'apprenant (stratégies communicatives d'apprentissage). 
21 L'alternance codique est un phénomène de contact apparaissant ordinairement lorsque plusieurs langues sont présentes. La réalité qu'offre le paysage linguistique algérien fait que l'alternance codique devient un phénomène fréquent, voire une stratégie naturelle de communication, lors des conversations quotidiennes des Algériens. Cette manifestation peut se définir, comme le propose J.-J. Gumperz (1989) comme étant «la juxtaposition à l'intérieur d'un même échange verbal de passages où le discours appartient à deux systèmes ou sous-systèmes grammaticaux différents » (p. 57). Il peut donc s'agir de la juxtaposition de deux langues distinctes ; ainsi, on considère le changement de langue comme un fait d'alternance codique.

Pour sa part, M. Causa (2002) voit l'alternance codique comme « les passages dynamiques d'une langue à l'autre dans la même interaction verbale» (p. 2). On peut donc aisément supposer qu'il y a effectivement alternance codique lorsqu'un locuteur fait alterner deux langues (au moins) dans un même discours. Plusieurs raisons peuvent être avancées quant à l'usage de ce phénomène. Pour certains (Hamers \& Blanc, 1983, Causa, 2002, etc.), l'alternance codique peut être un indice d'incompétence de l'apprenant en langue cible mais aussi un phénomène positif (Cambra Giné, 2003) et permet le recours à des stratégies compensatoires entre les langues connues de l'apprenant (Causa, 2002). Pour l'apprenant de LE, la LM constitue un point de repère fondamental.

Dans une perspective interactionniste, l'alternance codique est vue comme une stratégie de communication. L'utilisation de la LM en classe de LE a été considérée comme un recours mais aussi comme un interdit. Cette question de la LM a déjà été posée par les différentes méthodologies c'est-à-dire que selon la méthode d'enseignement préconisée, l'utilisation de la LM est tolérée ou non. En effet, certaines méthodes (comme la méthode directe, par exemple) rejetaient toute intrusion de la LM lors d'un cours de langue. D'autres, au contraire (telle l'approche communicative) permettaient l'utilisation de la LM ; dans ce cas là, l'alternance n'est plus stigmatisée et, de ce fait, la tradition monolingue perd de son attrait.

Il en est de même au niveau des conceptions personnelles ou des choix pédagogiques d'un enseignant qui peut autoriser ou interdire ${ }^{13}$ à ses élèves d'avoir recours à leur LM (comme on pourra le constater dans l'analyse de notre corpus). Ce qui implique que dans certaines classes :

« [...], la communication fonctionne quasi exclusivement en langue cible alors que, dans d'autres, l'usage de la langue première ou langue de référence sera très largement toléré, voire même dans certains cas encouragé ; [...]. » (Castellotti, 2001 : 19)

Pour leur part, V. Castellotti (2001) ou M. Causa, $(1996,2002)$ voient dans l'alternance codique une ressource à mobiliser lors des interactions, une compétence à développer, voire même une richesse. Ce qui implique que parfois on peut attribuer à l'alternance codique le qualificatif de "stratégie communicative».

En ce sens, M. Causa $(1996,2002)$ pense que l'alternance codique doit être considérée comme une stratégie communicative à part entière parmi d'autres stratégies. A ce propos, cette auteure met l'accent sur le fait qu'enseignant et apprenant n'usent pas de stratégies communicatives pour les mêmes raisons. En effet, l'enseignant vise avant tout la transmission de connaissances et de savoir-faire en langue étrangère. Il peut recourir à cette stratégie pour expliquer, relancer l'interaction, pour garder l'attention ou le contact avec les élèves, etc. De son côté, l'élève utilise généralement cette stratégie lorsqu'il ne trouve pas les mots pour exprimer sa pensée. Ainsi, dans sa 
construction de la langue cible, il s'appuie sur sa langue source (LM) qui est sa pratique sociale de référence, son outil de communication principal. C'est la raison pour laquelle, M. Cambra Giné (2003) préconise de «considérer que la L1 est là, présente, faisant partie - qu'on le veuille ou pas - des répertoires, et que l'on ne peut pas évacuer, mais qu'il faut gérer en prenant certaines décisions » (pp. 248-249).

\section{Langue maternelle en classe de FLE}

Nous rappelons que, pour les élèves algériens, l'apprentissage du français se fait principalement dans un contexte formel. Ces derniers sont, dans leur majeure partie, confrontés d'abord à l'usage de l'arabe dialectal, puis de l'arabe classique, ensuite de la langue française (au primaire) et de la langue anglaise (au collège). Nous notons que dans ces langues en présence, la langue maternelle se trouve en première position. $\mathrm{Ce}$ contact entre plusieurs langues risque d'engendrer le mélange de ces langues.

Dans cette contribution, nous nous posons la question de savoir comment on peut expliquer ces changements de langue opérés en classe de FLE. L'alternance codique peut être initiée soit par l'élève, soit par l'enseignant.

\section{Du côté de l'élève}

Nous avons interviewé cinq élèves de première année secondaire (trois garçons : $E^{14} 1$, E2 et E3 et deux filles : E4 et E5).

- A la question : quelle langue utilises-tu dans ta vie quotidienne? (après une pause moyenne, nous avons réitéré notre question) ma loughatahderbihakoulyoum? (quelle langue parles-tu tous les jours?).

Les cinq élèves ont répondu, sans hésitation, « l'arbia » c'est-à-dire le dialecte.

- A la question : tahder (parles-tu) en français pendant le cours de français?

Un seul élève (E2) avoue ne pas parler du tout (que ce soit en français ou en arabe, même si l'enseignant l'interroge. Les autres répondent soit par l'affirmative (E1) soit par un peu (E3, E4, E5).

- A la question : parles-tu en arabe 'fi wast' (dans) la classe?

- E1 :'pas du tout je parle pas l'arabe puisque c'est un cours de français ${ }^{15}$.

- E4: 'je demande à la maîtresse en français quand je sais le dire ou je le demande bel'arbia (en arabe - stratégie de substitution) ou bien je prends le dictionnaire (stratégie de réalisation).

- E3et E5, quant à eux, disent avoir volontiers recours à l'arabe dialectal.

- E2 n'a pas voulu répondre.

- A la question : à quel moment ou quand utilises-tu la langue arabe en cours de français?

- E3 : 'surtout 'quand rani (je suis) coincé'

- E5 : 'je parle arabe mieux que pas répondre'

- Pour ces deux derniers élèves, on remarquera qu'ils font le choix d'inclure leur LM dans leur discours pour donner une réponse à l'enseignant ; leur objectif étant de prendre la parole.

- E4 :'quand je connais pas les mots' 
stratégie de substitution utilisée par ces trois élèves leur permet d'avoir recours momentanément à la LM pour résoudre un problème ${ }^{16}$. Ce qui permet de faire avancer la communication en classe de langue.

- A la question: kifechdirkiikounou (comment fais tu lorsqu'il y a) des problèmes en langue française quand tu es bloqué ?

- E1 répond : - quand je suis bloqué, je réfléchis et puis d'abord (en haussant les épaules) pas du tout je parle pas l'arabe puisque c'est un cours de français.

Dans ce cas là, on pourra remarquer que le lieu est un facteur déterminant (Queffélec, 2002). En effet, la classe de langue est un espace où la contrainte institutionnelle est forte en ce sens où elle peut déterminer chez certains le choix d'une seule langue, ici la langue étudiée, en l'occurrence, le français.

- E3 'surtout quand rani (je suis) coincé'

- E5 'je parle arabe mieux que pas répondre'

Pour ces deux derniers élèves, on remarquera qu'ils incluent leur LM dans leur discours pour donner une réponse à l'enseignant. L'essentiel étant de prendre la parole en classe.

- E4 : 'manah'dar bel arbia ${ }^{17}$ parce que la maîtresse matrelinachnahadrou en arabe (elle ne nous laisse pas parler en arabe) eh ben je parle en 'arabe de l'école' comme ça ma zeguichaliya' (elle ne me gronde pas).

On notera ici que l'élève n'a apparemment pas conscience que pour cette enseignante, même le recours à "l'arabe de l'école », c'est-à-dire l'arabe standard est prohibé. Nous comprenons par là que cet élève pense que la maitresse leur interdit uniquement d'avoir recours à l'arabe algérien sous peine "de se faire gronder». Pour celui-ci, le fait de parler en arabe standard est une référence à la norme et peut être donc permis.

- A la question : 3 lechtahderbe l'arbia (pourquoi tu parles en arabe) en classe ?

- E3 : 'comme ça nejmounehadrou (on peut parler)'

- E4 : 'bechmankounch (pour ne pas être) en panne'

Le choix de la LM se justifie par le fait qu'il bloque sur un mot, une expression; le dire en arabe dialectal ou en arabe standard permet de ne pas rester en panne. Ce qui permet donc de débloquer la communication. Ceci incite aussi les élèves à prendre la parole en classe. Ainsi, l'alternance possède une visée communicative et devient un mode de communication privilégié par les élèves.

(ésumé, on retient que trois élèves (E3, E4 et E5) sur cinq ont recours à leur LM. E1 utilise uniquement la langue française en cours de FLE et E2 ne prend pratiquement pas la parole. E3, E4 et E5 motivent leur recours à l'alternance codique principalement dans des moments, dirons-nous de " détresse verbale » (Causa, 2002) c'est-à-dire lorsqu'ils veulent dire quelque chose alors que les mots leur manquent pour l'exprimer.

C'est une alternance codique à fonction métalinguistique. Le changement de code est un moyen pour s'exprimer face au manque de vocabulaire. Cet appel à l'aide rejoint ainsi la catégorie des stratégies d'apprentissage (Causa, 2002).

Ainsi, l'élève a souvent recours à sa LM pour résoudre un problème communicatif. Notons que l'élève, conscient des difficultés qu'il a à surmonter, essaie de les dépasser. C'est en ce sens que C. Faerch et G. Kasper ont défini les stratégies comme étant des " plans potentiellement conscients » (cités par P. Bange, 1992). 


\section{Du côté de l"enseignant}

Pour mener à bien ses objectifs, l'enseignant utilise différentes stratégies dont l'utilisation de la LM en classe de langue. Pour en connaître le pourquoi et le comment, nous avons fait une enquête auprès de cinq enseignants. Quatre enseignants sur les cinq enseignants interrogés enseignent, depuis au moins cinq ans, dans le secondaire ; le cinquième, quant à lui, enseigne la langue française depuis plus d'une dizaine d'années.

Sur les cinq, un seul n'autorise absolument pas l'utilisation de la LM en classe ${ }^{18}$ pour lui, il est strictement interdit de parler en arabe dans sa classe sinon pourquoi enseigner la langue française.

2 Par contre, un autre accepte de traduire littéralement et pratiquement tout à ses élèves. Ce dernier justifiant cela par le fait qu'il faut aider les élèves à mieux comprendre et donc de faire avancer son cours.

3 La troisième souligne que, lorsqu'elle se trouve dans des situations d'incompréhension de la part des élèves, elle utilise "malheureusement » la LM en cours de FLE, mais cela après avoir eu recours à d'autres possibilités comme l'image, la gestuelle, etc.

Les deux autres nous disent avoir recours à la LM surtout dans les séquences de vocabulaire (principalement pour des notions abstraites, pour lesquelles le recours à l'image ou la mimique est impossible) ou pour des leçons de grammaire. L'un des deux nous dira qu'il devait simplifier les leçons (en utilisant la LM) pour qu'elle soit comprise. Il rajoute que si les élèves ne comprennent pas la leçon, ils risquent de se désintéresser du cours. Les enseignants ayant recours à la LM le font aussi pour rétablir l'ordre dans la classe. Ils rajoutent le fait que les élèves n'ont pas tous le même niveau en langue et qu'ils se voient dans l'obligation de parler en arabe car c'est la seule issue pour débloquer une situation qui gêne et risque de retarder le cours.

Néanmoins, à la question, "autorisez-vous les élèves à utiliser leur LM ? ", ces mêmes enseignants répondent oui, mais dans une certaine mesure. Autrement dit, l'enseignant utilise la LM avec modération, selon les cas.

Généralement, la séquence de vocabulaire est celle où l'alternance codique a le plus souvent lieu. Ces enseignants alternent la langue pour faciliter l'intercompréhension. L'arabe aide donc à la compréhension du français et par là peut faire avancer l'interaction.

4 Nous avons posé aussi la question suivante : « lorsque vous utilisez la LM, vous le faites pour quelles raisons?».

Trois enseignants répondent que c'est surtout pour des questions de discipline. Ils utilisent aussi la LM lorsqu'il est question de vocabulaire ou pour donner des explications (stratégie contrastive) mais aussi parfois quand le sujet touche au vécu des apprenants, à leur vie quotidienne. Le quatrième enseignant avoue avoir recours à la LM de façon machinale et souligne le fait qu'il pense qu'en utilisant la LM les élèves comprennent mieux.

9 L'alternance codique peut aussi être choisie pour gagner du temps car trois enseignants sur cinq estiment que c'est une manière rapide et efficace pour poursuivre leur cours.

D'une manière générale, l'enseignant utilise la LM commune surtout selon l'objectif de la séquence. 
51 Diverses raisons ont été évoquées : la volonté de se faire comprendre, pour rétablir l'ordre dans la classe, casser le mythe de la communication exolingue, etc. L'alternance codique peut s'expliquer aussi par l'envie de mettre l'apprenant en confiance et ainsi créer une relation de proximité avec les élèves, toujours dans un but particulier, celui de détendre l'atmosphère en classe avec comme finalité l'apprentissage de la LE.

Une dernière question portait sur le fait que nous avions remarqué que ce sont les enseignants les plus anciens qui refusent toute utilisation de la langue maternelle en classe de langue. La troisième enseignante nous dira qu'il est tout à fait normal, pour l'enseignant d'aujourd'hui, d'utiliser l'arabe en cours de FLE puisque l'élève n'est en contact avec la langue française pratiquement qu'en cours de FLE; ce qui n'était pas le cas à l'époque où les anciens enseignants assuraient leurs cours. En effet, dans les années 80 , les matières scientifiques étaient enseignées en langue française. Par conséquent, l'élève avait plus de contact avec la langue française.

Les constats énumérés ci-dessus l'ont été sur la base d'entretiens. Pour vérifier et compléter nos résultats, nous avons voulu savoir de quelle manière s'opère le recours à l'alternance codique; et ce, par le biais d'analyses d'interactions verbales. Notre recherche se base alors sur l'analyse du discours de l'enseignant et des élèves. Pour ce faire, nous avons demandé aux enseignants ${ }^{19}$ de bien vouloir nous laisser enregistrer des séances d'expression orale. Deux enseignants sur cinq seulement nous ont donné leur accord. Pour étudier l'alternance codique en tant que stratégie, nous le ferons sur la base de deux discussions dans deux classes différentes ( $\mathrm{C} 1$ et $\mathrm{C} 2$ ).

\section{L'alternance codique : une stratégie en classe de FLE}

L'alternance codique joue un rôle essentiel dans la construction du discours en classe de langue. Nous rappelons que M. Causa s'est intéressée à la question de l'alternance codique en classe de langue aussi bien du côté de l'enseignant que de celui de l'apprenant. Ses travaux ont montré que le recours à la LM peut s'expliquer en termes de stratégie. M. Cambra Giné (2003), quant à elle retient diverses fonctions de l'alternance codique : support à la compréhension et à la construction des énoncés par les apprenants ; mise en relief de l'activité métalangagière ; mise en relief de l'activité métacognitive ; mise en relief de l'activité relationnelle-affective.

D. More (1996) distingue les alternances codiques « relais ", qui fonctionnent comme des "bouées transcodiques» et permettant la poursuite de la communication, des alternances codiques « tremplin », qui elles, favorisent l'acquisition de la L2.

** Stratégie de substitution : c'est-à-dire des cas d'alternances qui fonctionnent comme des 'bouées' ayant comme fonction une demande d'aide à la formulation.

Exemple 1:

C1/TP002-E7 $7^{20}$ : euh ils veulent être libres les palestiniens euh euhkifechigouloubelikhasshoum issta3rfou bihoum (comment on dit qu'il faut qu'ils les reconnaissent).

E7 demande de l'aide face à une lacune lexicale (TP002). Les hésitations démontrent la « détresse lexicale » d'E7 qui cherche un appui et le recours à la LM a pour fonction la résolution d'un problème linguistique. 
L'alternance codique peut libérer des situations de blocage et par là peut constituer une stratégie de communication afin d'éviter la rupture de l'interaction, comme dans le cas suivant aussi où un élève initie l'alternance codique.

En effet, il recourt à la langue maternelle pour avoir des clarifications sur la différence entre un colloque et un séminaire :

Exemple 2:

C2/TP016-E4 : monsieur wachhouwaalfark bin colloque wa séminaire'/ (Quelle est la différence entre colloque et séminaire').

Dans les exemples 1 et 2, l'utilisation du dialecte ici a pour but de faciliter la communication. Il y a une volonté des élèves 7 (exemple 1) et 4 (exemple 2) à vouloir prendre et garder la parole; il s'agit donc d'une alternance relais qui favorise la communication entre les interactants.

$2{ }^{* *}$ Stratégie contrastive qui est «la mise en rapport explicite des deux langues en présence dans la classe pour en relever les points communs et/ou les différences " (Causa, 2002: 71). L'exemple ci-après montre la comparaison entre un système et un autre. L'enseignant a recours aux deux langues (LM et LE) ; dans ce cas, M. Causa (2002) parle de 'mise en correspondance bilingue'.

\section{Exemple 3:}

C2/TP008-P : oui bon alors dites-moi qu'aimeriez-vous faire à l'université' TP009-E13 : le droit

TP010-E2 : l'économie pour euh faire patron (rires) TP011-E10: moi je veux faire traduction

TP012-P : euh oui alors quand vous dites en français traduction euh en arabe ça correspond à tarjama

On retrouve aussi la fonction contrastive de l'alternance codique dans l'exemple 3 où l'enseignant (TP012) juxtapose de manière explicite la LM et la LE. Ce qui n'est pas sans rappeler la traduction interlinguale. Lorsqu'il a recours à la traduction d'un mot par exemple, cela permet à l'élève d'élargir son répertoire verbal. C'est ainsi que les stratégies de communication peuvent parfois rejoindre celles d'apprentissage.

5 Nous avons relevé, dans notre corpus, un autre exemple de la mise en correspondance de deux systèmes linguistiques et où l'enseignant demande une traduction en L2.

\section{Exemple 4:}

C1/TP013-P : c'est un héros + en arabe on dit TP014-E5 : el batal TP015-P : bien

67 L'exemple 4 montre que l'enseignant au TP013 veut s'assurer de la compréhension du mot «héros"; il demande donc aux élèves de le traduire. La traduction sert ici de procédé explicatif; traduction donnée par E5 en TP014 suivie de l'acceptation par l'enseignant (TP015).

8 En déployant la stratégie contrastive, l'enseignant génère « des ponts entre langue cible et langue maternelle » (Causa, $2002: 53$ ). Ces ponts permettent à l'élève d'effectuer des relations qui lui ouvrent la perspective de développer ses connaissances en langue cible. 

de répétitions et de reformulations ont été recensés.

71 - la répétition : la reprise non modifiée d'un élément énoncé précédemment (Vion, 1992). Il arrive que l'enseignant répète son propre discours (auto-répétition):

C1/TP005-P : oui mais comment imaginez-vous la vie des enfants palestiniens' TP006$\mathrm{E} 1$ : ils ont pas le droit de rester dans la palestine TP007-P : non mais euh + kifech tchoufou lichaa taa drari falestiniyin' (comment voyez-vous la vie des enfants palestiniens) (TP007).

C2/TP019-E1 : à l'université les cours c'est pas la même chose que que euh au lycée TP020-P : oui effectivement el dorossmachi kif kif (les cours c'est pas la même chose) pourquoi' compréhension (Causa, 1996). C'est donc une traduction qui peut servir à la compréhension et à la clarification du message. La fonction des répétitions ici est de faciliter la mémorisation par association à la LM (Causa, 1996).

Nous allons voir, ci-dessous, d'autres exemples tirés de notre corpus dans lesquels des élèves répètent en $\mathrm{LM}$ leur propre discours.

\section{Exemple 7 :}

C2/TP005 -E13 : pour étudier quelque chose de bien TP006 -P : oui TP007 -E13 : bech nekraa ajamliha.

Dans l'exemple 7, suite à la réponse de l'enseignant 'oui' (TP006), E13 répète en LM (TP007) ce qu'il a énoncé en TP005. E13 a probablement interprété le 'oui' de l'enseignant (TP006) comme une demande de clarification de la part de l'enseignant. Dans cet exemple, nous remarquerons la capacité d'E13 à répéter en LM (TP007) son énoncé produit en LE au TP005. Ce qui peut être mis en relation avec le niveau avancé d'E13.

- la reformulation: c'est-à-dire une reprise avec modifications de propos tenus antérieurement (Vion, 1992).

80 Exemple 8:

C2/TP013 -E1 : je veux être enseignant plus tard pour donner des cours et pour partir à des colloques et euh/

TP014 -E5 : quoi' j'ai pas compris. 
TP015 -E1 : je veux dire je veux partir à des colloques euh ya3ni ijtima3 (c'est-à-dire réunion) pour rencontrer des personnes voilà.

Dans l'exemple ci-dessus, l'alternance codique sert aussi de support à la construction de l'énoncé d'E1 (Cambra Giné, 2003). En effet, au TP014, E5 interrompt E1 suite à l'incompréhension du mot colloque. E1 reformule au TP015 ses propos énoncés en TP013. Son intervention commence par un premier marqueur de reformulation paraphrastique (MRP) (Gülich et Kotschi, 1983) «je veux dire » et se poursuit avec le seconde MRP «ya3ni » (c'est-à-dire). L'interjection « euh » marque un temps d'arrêt pour pouvoir trouver le mot juste pour traduire colloque. Comme dans le cas des répétitions, la reformulation sert à la compréhension. Mais pour ne pas interrompre la dynamique discursive dans laquelle il se trouve, il fait le choix d'alterner les deux langues. Ce qui témoigne de sa compétence plurilingue.

Dans ce cas, il s'agit d'une alternance tremplin où l'élève clarifie le message par le biais de la reformulation en LM. En reformulant sa pensée en LM, E1 montre comment une même phrase peut être dite en LM et en LE.

Selon $\mathrm{M}$. Causa, la répétition et la reformulation jouent un rôle très important sur le plan communicatif mais aussi sur le plan acquisitionnel. Elles contribuent au développement de la compétence discursive en L2. De ce fait, l'alternance codique est considérée comme une stratégie à part entière aussi bien du côté de l'enseignant que de celui des élèves. Elle fait partie de ces stratégies d'acceptation de risques où l'enjeu communicatif est très important; elles sont donc positives parce qu'elles favorisent «l'épanouissement de la communication » (Bange, 1992).

L'utilisation de la LM en classe de FLE devient alors un mode particulier de communication $^{21}$. Au-delà du niveau relationnel, l'utilisation de stratégies communicatives peut, par conséquent, avoir un impact sur la communication. Par son attitude, l'élève essaie de maintenir le contact et ainsi il a la possibilité de débloquer, dirons-nous, la situation.

Ce "repli sur la L1 évite alors de s'exposer et de se retrouver sans défense " (Castellotti, 2001 : 16). Par son attitude, l'enseignant soutient les processus d'acquisition des apprenants. Il met donc en œuvre des stratégies en vue d'une transmission optimale des contenus d'enseignement de la langue cible. En cela, la notion de stratégie peut être mise en relation avec les notions de tutelle et d'étayage proposées par Bruner (1983).

86 En résumé, la plupart des enseignants ont recours à la LM. En alternant les langues en présence dans la classe, ces derniers offrent, par le biais de la première langue des apprenants, une meilleure compréhension. Le recours à la LM peut être bénéfique pour l'apprenant et c'est par ce biais que les échanges sont plus dynamiques.

87 L'alternance codique, en tant que stratégie utilisée en classe de FLE, peut fonctionner comme « relais » afin de poursuivre la communication mais aussi comme "tremplin » en ce que la LM sert d'appui pour la construction du sens et pour l'appropriation linguistique. Les élèves alternent donc les langues pour apprendre et communiquer.

Globalement, l'intercompréhension permet de justifier l'utilisation de stratégies en vue de favoriser mais surtout de stimuler la communication en classe. Nous avons vu que l'utilisation de stratégies par les acteurs de la classe s'opère principalement dans le but d'anticiper un problème de communication. La raison principale évoquée par les élèves, quant au recours de la LM, est le déficit lexical. 
89 En conclusion, nous pouvons dire que notre analyse du discours scolaire nous a permis de faire le point sur la manière dont l'alternance codique est mise en œuvre en classe de FLE. Les résultats de notre étude mettent en avant l'alternance codique comme source de richesse ; elle devient alors ce «facilitateur » vers la compétence langagière, un outil pour la communication et l'apprentissage de la L2. En conséquence, la réalité plurilingue de la classe de FLE doit nous inciter à prendre en compte le phénomène du recours à la $\mathrm{LM}$, non plus comme un élément négatif mais de le considérer comme ressource pour apprendre et communiquer (Cambra Giné, 2003), comme une libération de l'expression et comme reconnaissance de l'apprenant en tant qu'être plurilingue.

Il est donc nécessaire de prendre en compte l'importance d'une approche plurilingue pour gérer au mieux l'enseignement/apprentissage d'une langue. Néanmoins, même si nous jugeons que le recours à la L1 est utile en classe de FLE, nous pensons qu'il faut en faire un bon usage et faire le choix d'une "pratique raisonnée " de l'alternance codique. En ce sens, il faudrait peut-être s'intéresser (entre autres) au problème de la formation des enseignants.

\section{BIBLIOGRAPHIE}

BANGE P., «A propos de la communication et de l'apprentissage de L2 notamment dans ses formes institutionnelles », in AILE, D. Veronique (dir.), Nouvelles perspectives dans l'étude de l'apprentissage d'une langue étrangère en milieu scolaire et en milieu social, $\mathrm{n}^{\circ} 1,1992, \mathrm{pp} .53-85$.

CAMBRA GINE M., Une approche ethnographique de la classe de langue, Paris, Didier collection, LAL, 2003.

CASTELLOTTI V., « Pour une perspective plurilingue sur l'apprentissage et l'enseignement des langues », in V. Castellotti (dir.), D’une langue à d'autres : pratiques et représentations, Publications de l'université de Rouen, collection Dyalang, 2001, pp 9-38.

CAUSA M., L'alternance codique dans l'enseignement d'une langue étrangère. Stratégies d'enseignement bilingues et transmission de savoirs en langue étrangère, Bruxelles, Peter Lang, 2002.

CAUSA M., «L'alternance codique dans le discours de l'enseignant. Entre transmission de connaissances et interaction ", in F. Cicurel \& E. Blondel, (dirs.), La construction interactive des discours de la classe de langue, Les carnets du Cediscor, nº 4, 1996, pp. 111-129.

DABENE L., Repères sociolinguistiques pour l'enseignement des langues, Paris, Hachette, 1994.

GRANDGUILLAUME G., Arabisation et politique linguistique au Maghreb, Paris, édition Maisonneuve et Larose, 1983.

GULICH E., KOTSCHI T., «Les marqueurs de la reformulation paraphrastique » in Cahiers de linguistique française, $\mathrm{n}^{\circ} 5$, Connecteurs pragmatiques et structure du discours, Université de Genève, 1983, pp. 305-351.

LUDI G. et PY B., Etre bilingue, Berne, Peter lang, 2003. 
MOORE D., « Bouées transcodiques en situation immersive ou comment interagir avec deux langues quand on apprend une langue à l'école », in S. Oeschet, B. Py (dir.), Le bilinguisme, AILE, $\mathrm{n}^{\circ}$ 7, 1996, pp. 95-121.

PORQUIER R., PY B., Apprentissage d'une langue étrangère : contexte et discours, Paris, Crédif, 2004.

QUEFFELEC A., et al., Le français en Algérie. Lexique et dynamique des langues, Bruxelles, Duculot, 2002.

TALEB IBRAHIMI Khaoula, Les algériens et leurs langues, éléments pour une approche sociolinguistique de la société algérienne, Alger, ed. El Hikma, 1997.

VION R., La communication verbale. Analyse des interactions, Paris, Hachette, 1992. VYGOTSKI L., Pensée et langage, Paris, Éditions sociales, 1985.

\section{NOTES}

1. Bien que cette notion soit très ambigüe (cf. Dabène, 1994), nous entendons par LM, celle apprise par le sujet dans son milieu familial ou bien celle de sa première enfance. En ce sens, on peut aussi parler de langue 1 (L1) qui désigne la langue de première socialisation. Ici, la L1 des élèves et des enseignants est l'arabe dialectal ou 'darija'.

2. Ce sont, généralement, les enseignants les plus anciens, formés eux-mêmes par des enseignants qui ont été le " produit » des méthodologies directes, qui refusent l'utilisation de la langue maternelle en classe de FLE.

3. Nous notons la variété des termes utilisés pour désigner l'alternance codique (code-switching, changement de code, etc.). Dans cette étude, nous ne ferons pas de différence entre ces termes.

4. Les données consistent en des interactions de deux classes de terminale en cours de FLE (avec respectivement 20 et 25 élèves) enregistrées (à l'aide d'un dictaphone car nous n'avions pas eu d'autorisation pour faire des enregistrements vidéo) et transcrites.

5. A la fin du cycle secondaire, l'élève doit devenir un utilisateur autonome du français. Donc, les élèves de notre enquête étaient censés maîtriser cette langue.

6. Pour plus de détail sur la désignation 'langue arabe', voir A. Queffélec (2002:33).

7. En effet, de sa naissance à sa première année de scolarisation, l'élève algérien n'entend, puis ne parle que sa langue maternelle, c'est-à-dire soit l'arabe algérien, soit le berbère. Ce qui fait de l'arabe classique une langue seconde à acquérir pour les élèves, puisque son apprentissage ne débute qu'à partir de la première année de scolarisation (première année primaire).

8. C'est-à-dire l'arabe algérien ou le berbère, selon la région dans laquelle on se trouve.

9. Variantes qui n'altèrent toutefois pas l'intercompréhension entre les locuteurs de ces régions.

10. Pour D. Moore (2001), les marques transcodiques "désignent toute trace discursive, perçue et traitée comme telle, de la présence d'une deuxième langue dans le répertoire verbal » (p.77).

11. Dans le cadre de cet article, nous nous intéressons particulièrement à l'alternance codique.

12. La conversation exolingue implique une conversation entre natifs et non-natifs. Elle peut aussi être définie par l'asymétrie et la divergence entre les répertoires linguistiques des participants (Lüdi \& Py, 2003). Ainsi, bien que les enseignants et les apprenants de notre corpus possèdent la même LM, nous considérons l'enseignant comme un locuteur natif pour sa maîtrise de la LE, et l'apprenant comme le locuteur non-natif. Pour parvenir à une communication efficace, ces derniers doivent pouvoir gérer le déséquilibre dans leurs compétences langagières.

13. Dans ce cas, l'enseignant soutient l'existence d'un « contrat codique » (Cambra-Giné, 2003).

14. $\mathrm{E}$ = élève. Nous avons choisi d'alterner langue française et dialecte dans nos questions afin de mettre à l'aise les élèves ; ce qui n'a pas été le cas avec les enseignants.

15. Les réponses des élèves sont en gras. 
16. Pour résoudre ces problèmes, les élèves font appel à des stratégies de communication.

17. L'élève désigne ici le dialecte.

18. C'est celui qui possède plus de dix années d'expérience (voir note de bas de page $\mathrm{n}^{\circ} 75$ ).

19. Ce sont ceux sollicités pour l'entretien.

20. $\mathrm{C} 1$ : correspond à la classe 1 dont la discussion portait sur la vie des enfants en Palestine ; C2 : correspond à la deuxième classe dont la discussion portait sur les études à l'université.

$\mathrm{TP}$ : correspond au tour de parole (ici le quinzième dans l'interaction verbale); E7 est l'élève à qui nous avons attribué le numéro 7. P correspond à l'enseignant.

Conventions de transcription : +,++,+++ : pause très brève, brève, moyenne.

': Intonation montante - / Interruption

21. Certes, il y a certainement d'autres raisons, comme le souligne Georges Lüdi (1999) : chercher à connaître l' " effectif potentiel acquisitionnel ", en d'autres termes, voir s'il existe des effets positifs ou non dans le processus d'apprentissage.

\section{RÉSUMÉS}

La situation sociolinguistique algérienne se caractérise par la présence de plusieurs langues. Cette diversité langagière engendre l'apparition de nombreux phénomènes (tels que les emprunts, l'alternance codique, etc.) que l'on retrouve aussi dans des interactions didactiques. Aussi, interrogerons-nous, dans cet article, sur la place et le rôle de la langue maternelle en classe de français langue étrangère en Algérie ; ceci à partir d'interactions en cours de français et d'entretiens avec des enseignants et des élèves du secondaire. A cet effet, nous voudrions vérifier l'idée selon laquelle la mise en œuvre de stratégies (en particulier le recours à la langue maternelle) est nécessaire à la fois à l'intercompréhension et au déblocage de la communication en classe de français langue étrangère.

The Algerian sociolinguistic situation is characterized by a variety of existing languages. This linguistic diversity led consequently to the emergence of many phenomena (such as borrowing, code-switching, etc.) mainly observed in educational interactions. So, an important question is posed in this article about the position and the role of the mother tongue in French as a foreign language in the Algerian context; in interactions and interviews with both secondary teachers and students. To this end, we would like to test the idea that the implementation of strategies (the use of mother tongue in particular) is necessary both to mutual understanding and releasing communication in an FFL classroom.

\section{INDEX}

Keywords : mother tongue, French as a foreign language, code switching, strategy, communication

Mots-clés : langue maternelle, français langue étrangère, alternance codique, stratégie, communication 


\section{AUTEUR}

\section{RABEAA BENAMAR}

Université Abou Bekr Belkaid - Laboratoire DYLANDIMED - Tlemcen - Algérie

BENAMAR Rabéa est maître de conférences en didactique des langues, à la faculté des lettres et des langues de l'Université Aboubekr Belkaid (Tlemcen). Elle est membre du laboratoire de recherches en « Dynamiques des langues et Discours en Méditerranée » (DYLANDIMED - U. Tlemcen). Elle est titulaire d'une thèse de doctorat, intitulée « Revalorisation de l'oral en classe de FLE au département des lettres arabes (Tlemcen) et son apport dans les interactions comme véhicule des apprentissages ", et auteur de publications telles que «L'oral dans l'écrit des étudiants. Analyse des marques récurrentes » (in Resolang $n^{\circ}$ 2, Décembre 2007), et « Stratégies d'aide à la production orale en classe de FLE » (in Synergies Algérie, $n^{\circ} 8,2009$ ). 